FERMILAB-SLIDES-20-077-V

\title{
Energy calibration of the ProtoDUNE-SP TPC
}

\section{Richie Diurba (University of Minnesota) for the DUNE Collaboration ICHEP 2020}




\section{$\mathrm{dE} / \mathrm{dx}$ in ProtoDUNE-SP}

- Precise $\mathrm{dE} / \mathrm{dx}$ measurements needed to make cross-section and Bragg peak measurements for pions, protons, muons, and kaons in ProtoDUNE.

- Use Modified Box Model

- Formula pioneered by ICARUS that is a modification of Birks' Law

$\left(\frac{d E}{d x}\right)_{\text {calibrated }}=\left(\exp \left(\frac{\left(\frac{d Q}{d x}\right)_{\text {calibrated }}}{C_{\text {cal }}} \frac{\beta^{\prime} W_{\text {ion }}}{\rho \mathscr{E}}\right)-\alpha\right)\left(\frac{\rho \mathscr{E}}{\beta^{\prime}}\right)$

$\mathrm{dQ} / \mathrm{dx}$ : Charge per step as reconstructed on the wires.

$\mathrm{C}_{\text {cal }}$ : Gain to convert from wire response (ADC*tick) to electrons.

$\xi$ : Local electric field

$\mathrm{W}_{\text {ion }}, \rho, \alpha, \beta$ ' are constants measured previously [1].

Measurements are needed for $\mathrm{C}_{\text {cal }}, \xi$, and $\mathrm{dQ} / \mathrm{dx}$ to calibrate $\mathrm{dE} / \mathrm{dx}$. 


\section{$\mathrm{dE} / \mathrm{dx}$ and Calibration}

- Calibration is done to go from ionized electrons to a quantifiable $\mathrm{dE} / \mathrm{dx}$.

Need to know the electric field

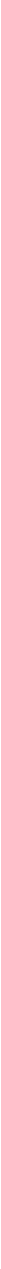




\section{Electric Field Calibration}

- Measure space charge effect

$\Delta E / E_{0}[\%]: Z_{\text {true }}=347 \mathrm{~cm}$ field distortions using positional distortions on the edges of the detector.

- Interpolate the electric field at a specific hit measured using the map of the electric field generated by the space charge effect calibration.

- The $\mathrm{dE} / \mathrm{dx}$ measurement also corrects for the squeezing and stretching of the step length (dx) using the space charge effect distortion measurements.

- The nominal electric field was measured at $0.487 \mathrm{kV} / \mathrm{cm}$
See previous talk by M. Mooney
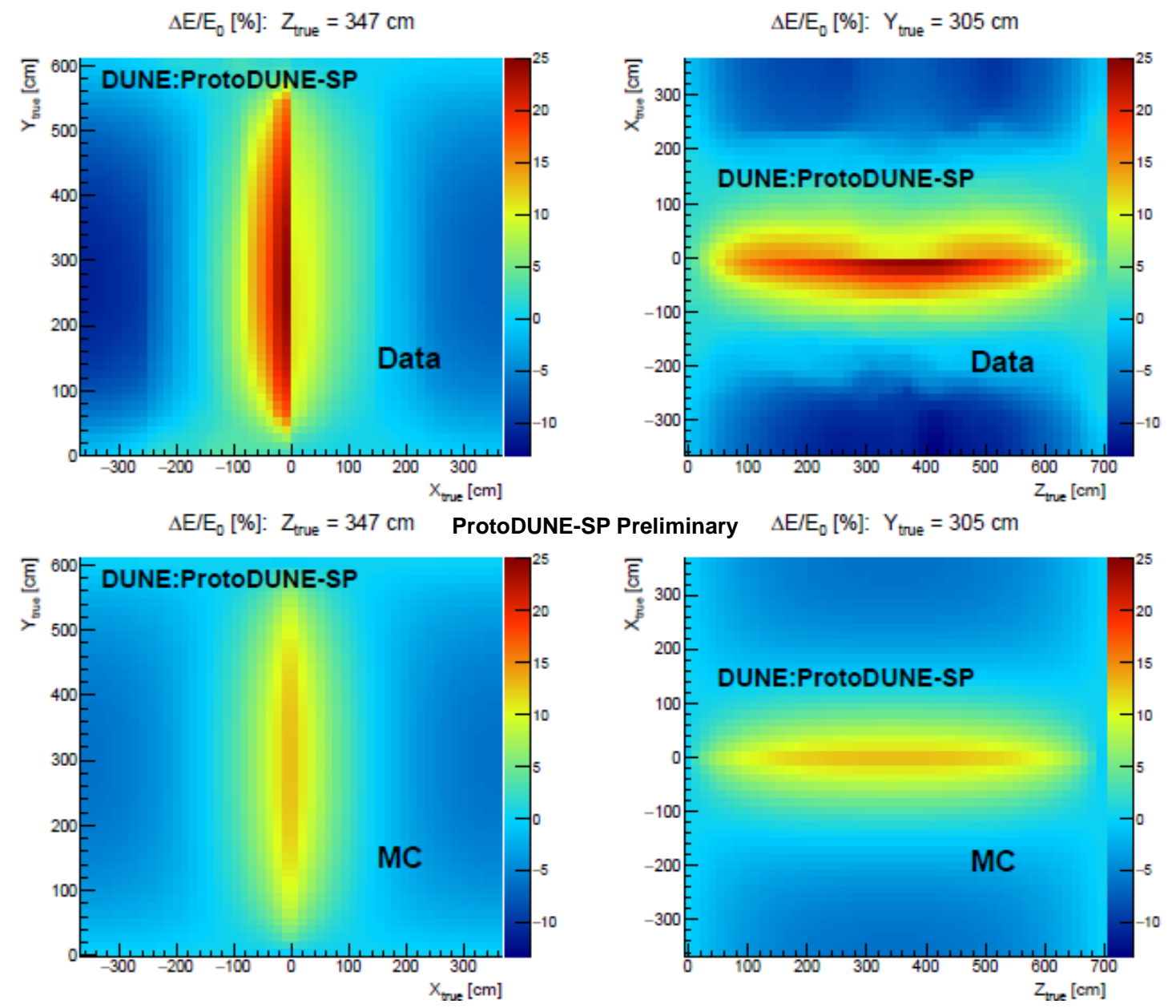

Electric field deviations measured using the end points of cathode-crossing tracks. 


\section{dQ/dx Calibration}

- Use cosmic muons at high residual range as they have well-defined $\mathrm{dE} / \mathrm{dx}$ that is known theoretically by less than $1 \%$ uncertainty using the Landau-Vavilov theory [2].

Calibrating $\mathrm{dQ} / \mathrm{dx}$ :

1. Select cathode-crossing cosmic tracks enter and exit detector.

2. Cut out cosmic muons that have certain track angles to avoid geometrical effects. (Cut if $65^{0}<\theta x z<110^{0}$ or $70^{\circ}<\theta y z<110^{\circ}$ )

3. Measure fluctuations in $d Q / d x$ as a function of $Y Z$. Then Measure $\mathrm{dQ} / \mathrm{dx}$ as a function of drift distance $(\mathrm{X})$.

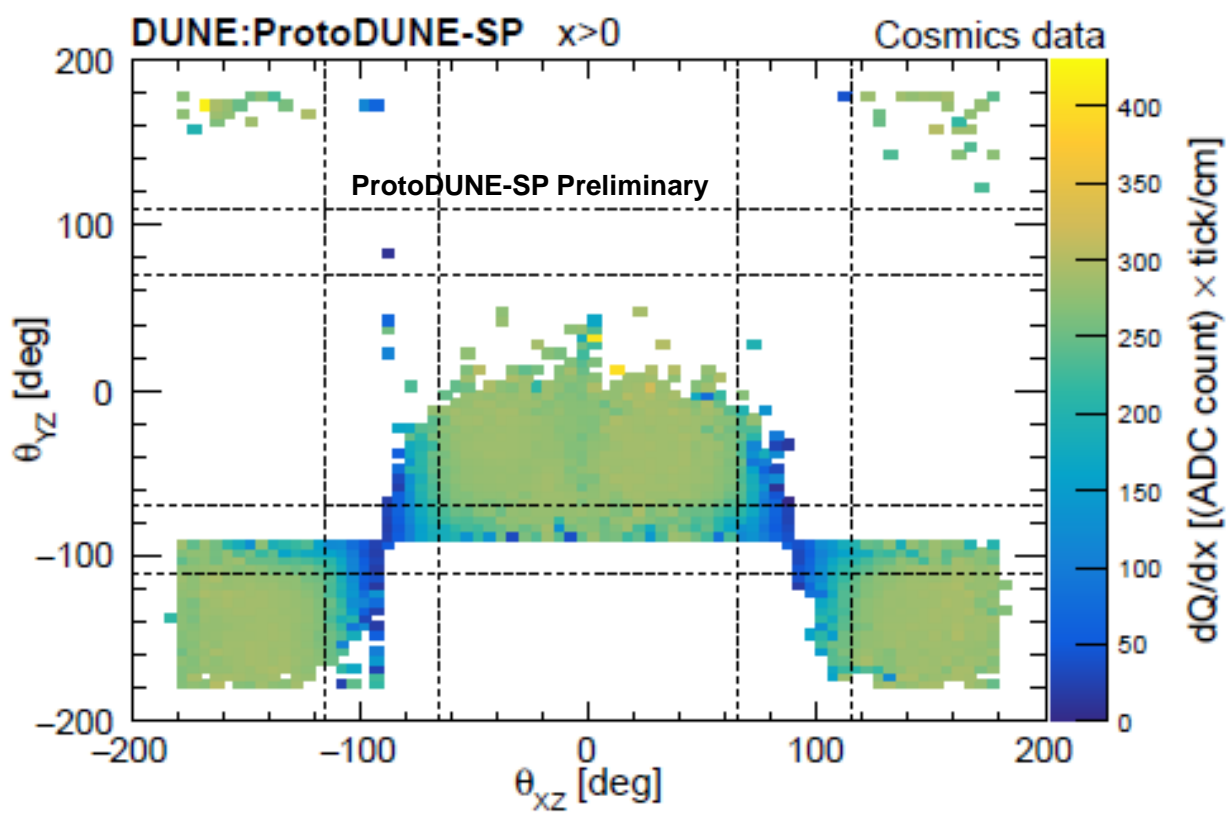

Track angles of cosmic muons for the non-beam side drift volume. The cathode plane of the detector sits at $\mathrm{X}=0$.

4. Normalize $\mathrm{dQ} / \mathrm{dx}$ between $\mathrm{drift}$ volumes. 


\section{XYZ Calibration of $d Q / d x$}

1. $Y Z$ calibration

- $\mathrm{C}(\mathrm{y}, \mathrm{z})=\frac{d Q / d x_{\text {global } y z}}{d Q / d x(Y, Z)}$

2. X Calibration

- $\mathrm{C}(\mathrm{x})=\frac{d Q / d x_{\text {global } x}}{d Q / d x(X)}$

3. Normalization

- $\mathrm{N}_{\mathrm{Q}}=\frac{d Q / d x(\text { anode })}{d Q / d x_{\text {global }}}$

4. Full $\mathrm{dQ} / \mathrm{dx}$ calibration

- $\mathrm{dQ} / \mathrm{dx} \mathrm{cal}_{\text {cal }}=C(x) C(y, z) N_{Q} d Q / d x$

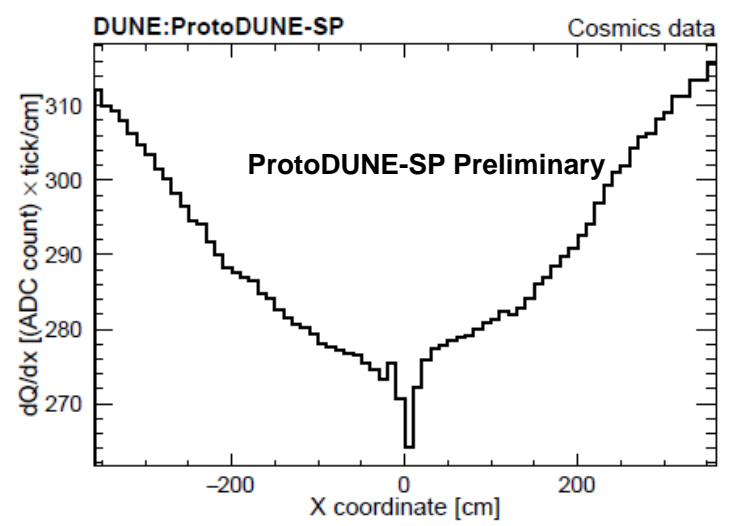

$d Q / d x$ as a function of $X$.
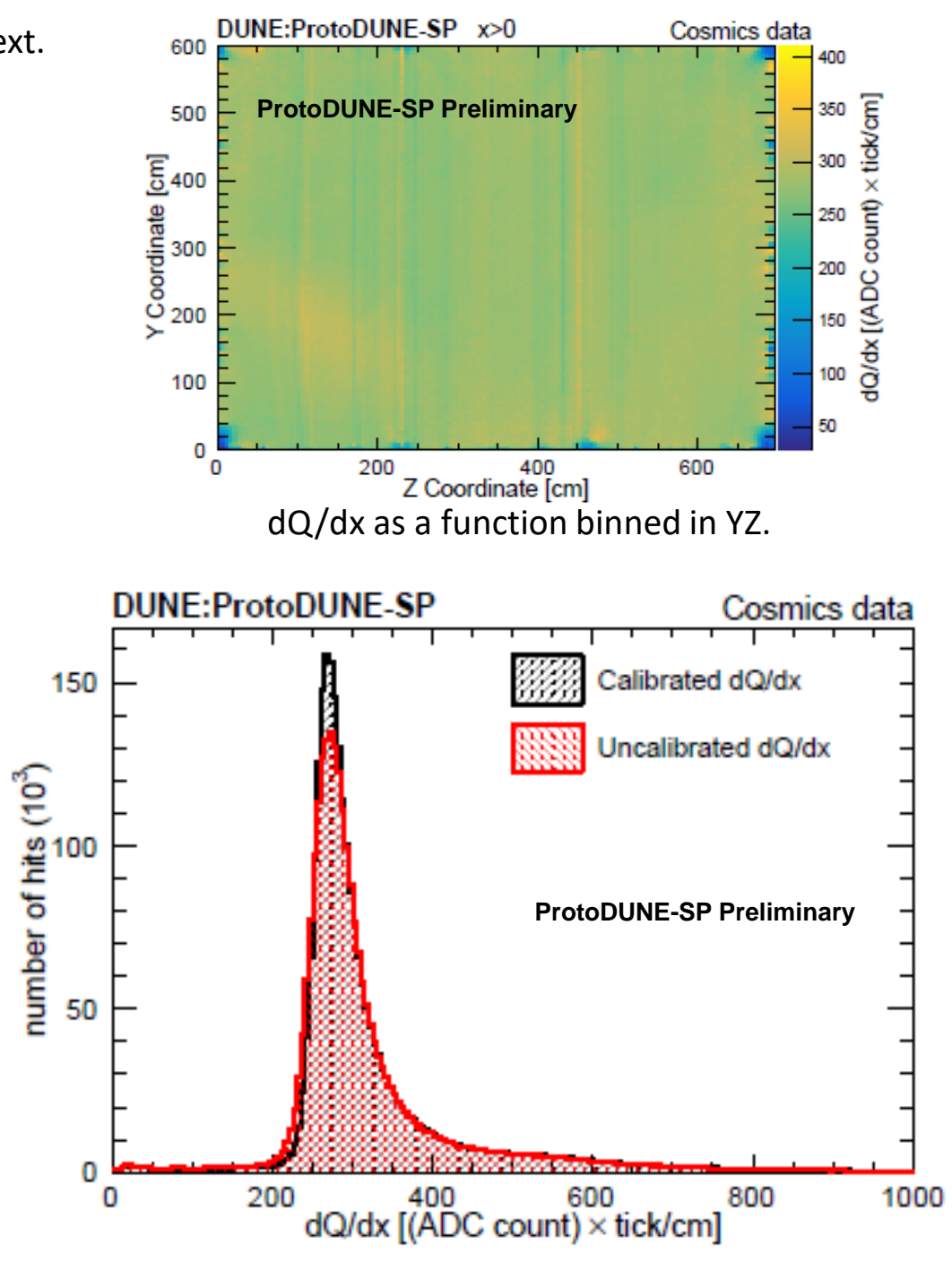

Final calibrated $d Q / d x$ 


\section{Energy Calibration $\left(\mathrm{C}_{\mathrm{cal}}\right)$}

Cathode-crossing tracks are again selected but with the following cuts:

1. Cut tracks that stop too close from the edges and with angles parallel to the wire plane.

2. Remove tracks that have reconstruction errors, such as broken tracks.

3. Use a $X^{2}$ optimization to measure $C_{c a l}$ using hits at high residual range $(120-200 \mathrm{~cm})$. Optimization is set that in a perfect detector $C_{\text {cal }}$ would be set to $5^{\star} 10^{-3}$ ADCxtick/e.

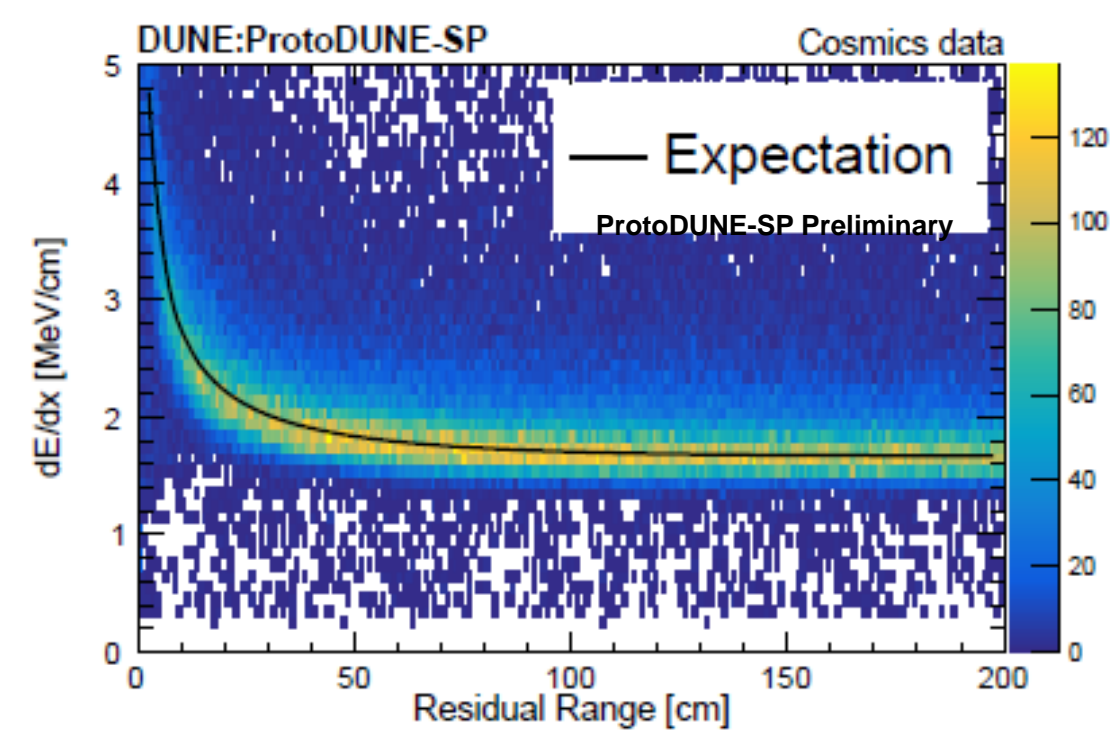

For this run: $\mathrm{C}_{\mathrm{cal}}=(5.4 \pm 0.1) * 10^{-3}$ ADCxtick/e High residual range area

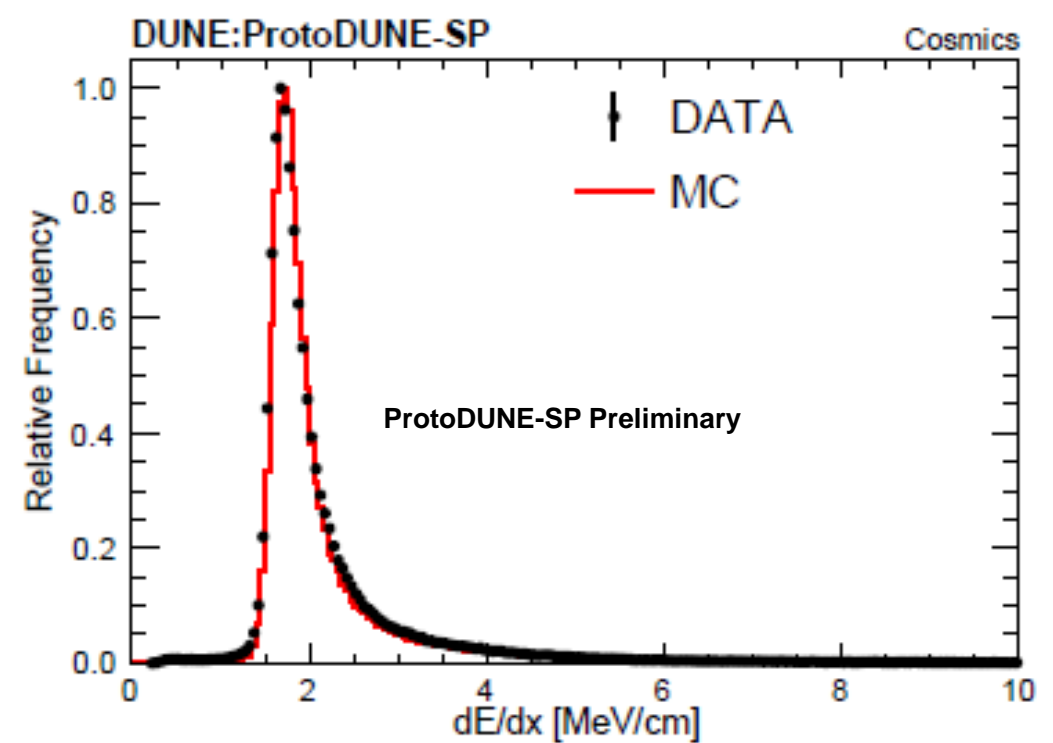

Calibrated $\mathrm{dE} / \mathrm{dx}$ for cosmic muons 


\section{Conclusion}

- Calibrations of $\mathrm{dE} / \mathrm{dx}$ were made by calibrating $\mathrm{dQ} / \mathrm{dx}$ across the detector and then scaling $\mathrm{dE} / \mathrm{dx}$ using the Landau-Vavilov theoretical $\mathrm{dE} / \mathrm{dx}$.

- Results included in ProtoDUNE-SP paper currently on the arXiv (arXiv:2007.06722).
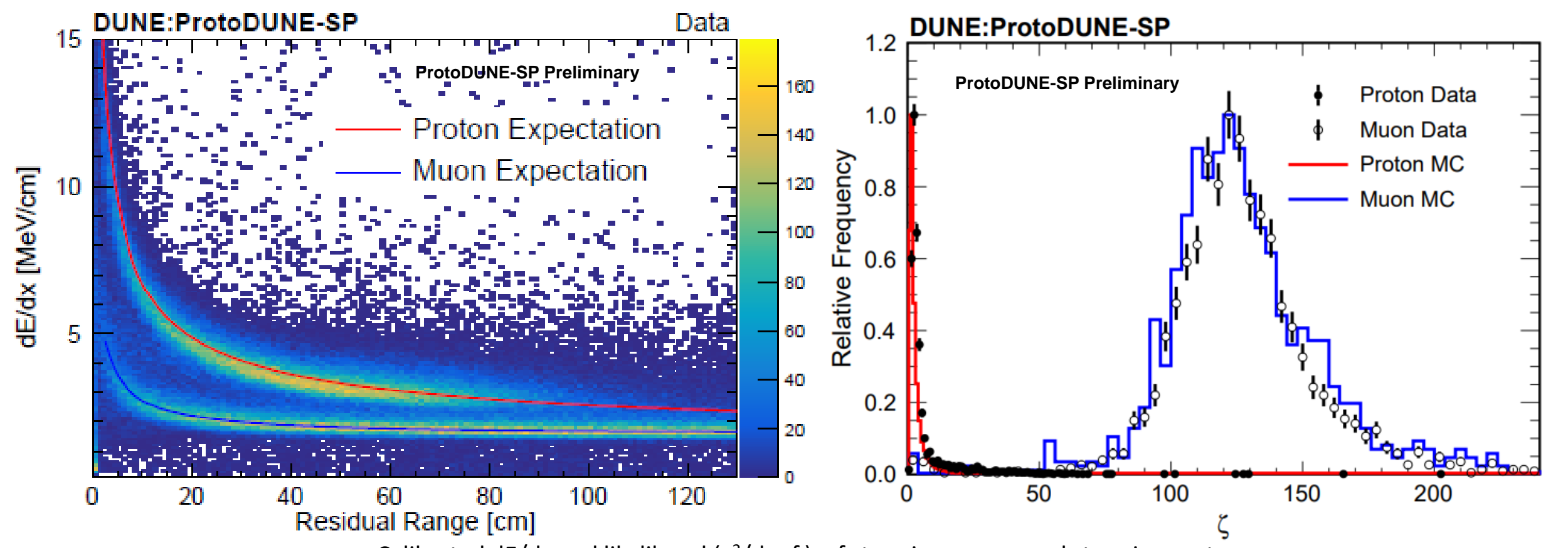

Calibrated $\mathrm{dE} / \mathrm{dx}$ and likelihood ( $\chi^{2} /$ d.o.f.) of stopping muons and stopping protons. 


\section{Backup Slides}




\section{Detector Basics}

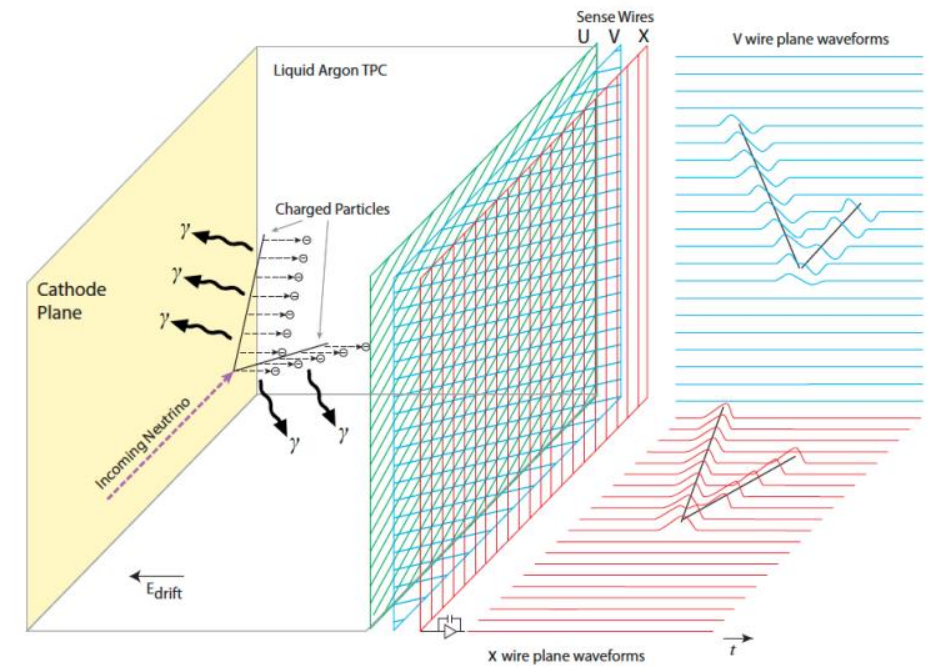

Demonstration of a LAr TPC reading the drift ionized electrons of a neutrino interaction [3].

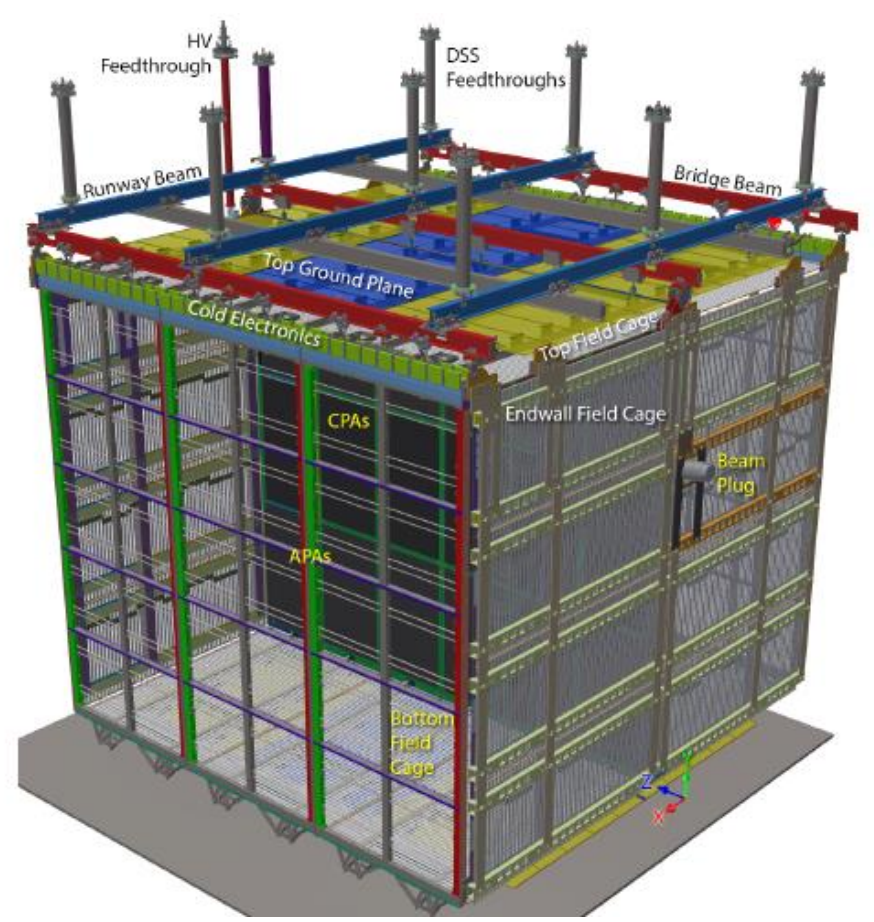

Diagram of the ProtoDUNE-SP detector. The $2^{\text {nd }}$ set of APAs is on the other wall of the detector.

[3] B. Abi, et. al., "Deep Underground Neutrino Experiment (DUNE) Far Detector Technical Detector Report Volume 1" arXiv:2002.02967, 2020. 


\section{$\mathrm{dE} / \mathrm{dx}$ in Monte Carlo}

Purity of Hits in MC using Cuts Outlined: $99.74 \%$

$\mathrm{C}_{\mathrm{cal}}=(5.03 \pm 0.01) * 10^{-3} \mathrm{ADCxtick} / \mathrm{e}$

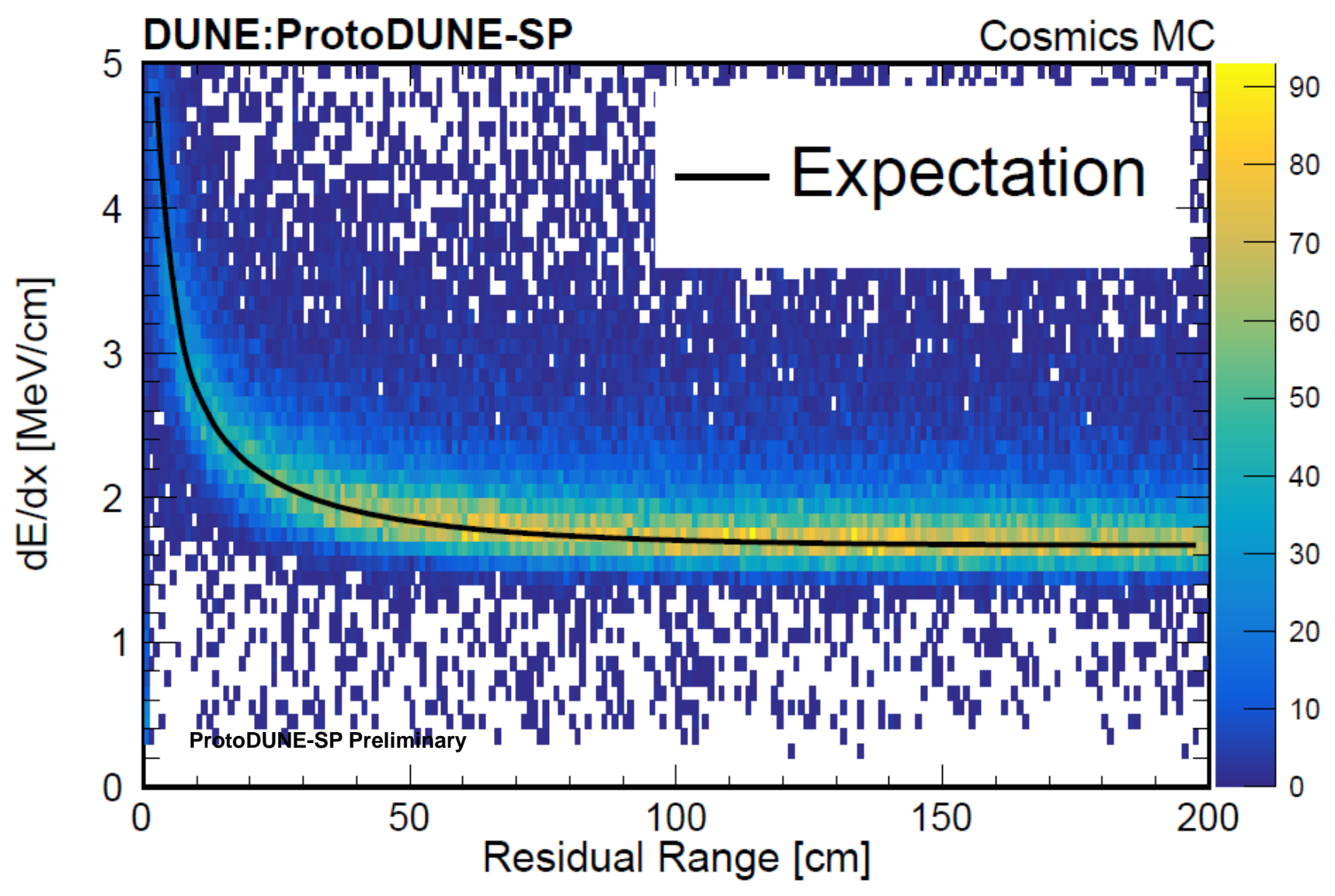

$\mathrm{dE} / \mathrm{dx}$ as a function of residual range for Monte Carlo 


\section{ADC Calibration}

- Measures the gain of the ADC to calibrate wire-to-wire differences in the readout.

- Uncertainty in the ADC calibration was considered to be at the few percent level.

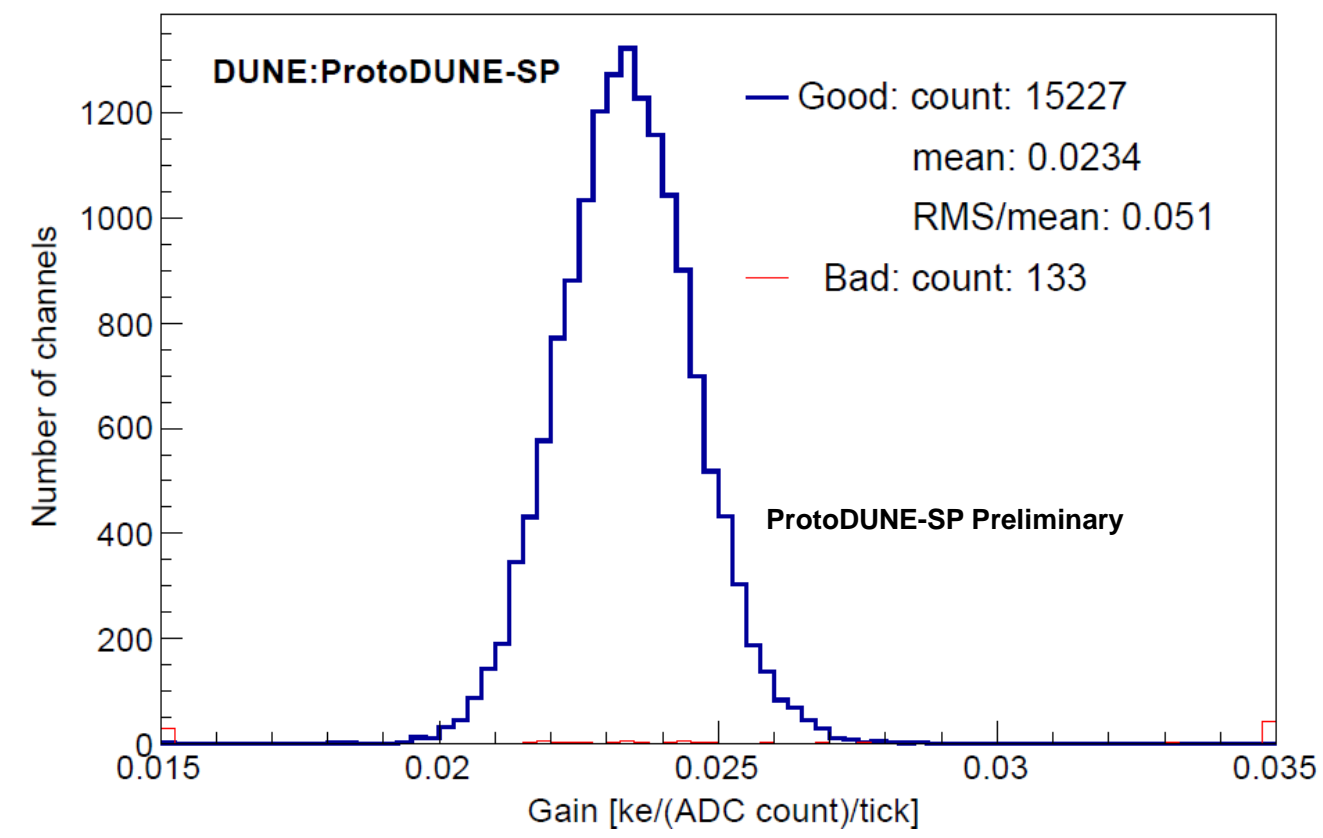

Measurements of the ADC gain using the pulser on the front end electronics. 